\title{
Research Paper: \\ The Effect of Filial Therapy on the Parenting Stress of Mothers of Children With Autism Spectrum Disorder
}

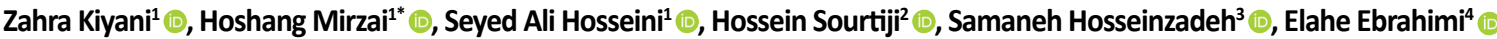

\author{
1. Department of Occupational Therapy, School of Rehabilitation, University of Social Welfare and Rehabilitation Sciences, Tehran, Iran. \\ 2. Department of Occupational Therapy, School of Rehabilition, Isfahan University of Medical Sciences, Isfahan, Iran. \\ 3. Department of Biostatistics, University of Social Welfare and Rehabilitation Sciences, Tehran, Iran. \\ 4. Department of Occupational Therapy, School of Rehabilitation, Shahid Beheshti Univercity of Medical Sciences, Tehran, Iran.
}

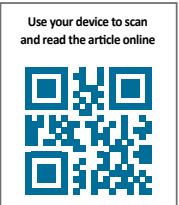

dteat on Kiyani Z, Mirzai H, Hosseini SA, Sourtiji H, Hosseinzadeh S, Ebrahimi E. [The Effect of Filial Therapy on the Parenting Stress of Mothers of Children With Autism Spectrum Disorder (Persian)]. Archives of Rehabilitation. 2020; 21(2):206-219. https://doi.org/10.32598/RJ.21.2.2726.1

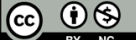

Received: 15 May 2018 Accepted: 30 Jun 2018 Available Online: $01 \mathrm{Jul} 2020$

Keywords:

Autism spectrum disorder, Parenting stress, Child-parent relationship, Play therapy

\section{A B STRACT}

Objective Autism Spectrum Disorder (ASD) is a neurodevelopmental disorder that affects the person' life. The prevalence of ASD in Iran is increasing. The complicated nature of this disorder disrupts the balance and work routines in the family, which puts a lot of stress on the entire family, especially mothers. Mental health of the mother affects all family members, including a child with ASD. On the other hand, mothers' confusion and failure in relation to their ASD child causes more stress and discomfort for them. In this study, we aimed to investigate the effect of filial therapy, as a play therapy, on the parenting stress of mothers with ASD children. Materials \& Methods In this experimental study, participants were the mothers of 32 children with ASD (9 girls and 23 boys) aged 4-12 years referred to autism centers and occupational therapy clinics in Isfahan, Iran who were selected using a convenience sampling method. After obtaining an ethical approval from the Research Ethics Committee of the University of Social Welfare and Rehabilitation Sciences (code: IR.USWR. REC.1396.97), and necessary permissions, researchers referred to the clinics and after explaining the purpose of the study to the mothers, an informed consent was obtained from them. Then, they completed a demographic form, Gilliam Autism Rating Scale-2 (GARS-2), and Abidin's Parenting Stress Index-Short Form (PSI-SF). Participants were randomly divided into two intervention and control groups after matching for the child's age, GARS score, and the education of children and mothers. The intervention group received 10 sessions of filial therapy, once a week, each for two hours, while the control group continued routine treatment. After intervention, PSI-SF was completed by all mothers again. The collected data were analyzed by using ANCOVA, paired t-test and independent t-test. Shapiro-Wilk test was used to determine the normality of PSI-SF data distribution at Pre-test and Post-test phases.

Results Paired t-test results showed that there was no statistically significant difference in the mean total parenting stress score between the two groups in the Pre-test phase $(P=0.679)$ but it was significant in the Post-test phase $(P=0.010)$. The results of independent $t$-test showed that the difference between the mean Pre-test and Post-test scores of total parenting stress in the control group was not significant $(P=0.268)$ but it was significant in the intervention group $(P=0.001)$. ANCOVA results showed that the Pre-test score of parenting stress had an effect on the total parenting stress score after intervention such that it could explain $82 \%$ of variations in this variable. After controlling the Pre-test score, the total parenting stress was significantly different in two groups $(\mathrm{P}=0.001)$. Eta squared value indicated that the intervention could explain $54 \%$ of changes in total parenting stress of mothers.

Conclusion Filial therapy can help better acceptance of the ASD children by their parents and reduce parenting stress by improving child-parent relationships. This method can be taught as a complementary intervention to the mothers of ASD children.

\section{* Corresponding Author:}

Hoshang Mirzai, PhD.

Address: Department of Occupational Therapy, School of Rehabilitation, University of Social Welfare and Rehabilitation Sciences, Tehran, Iran. Tel: +98 (912) 2208426

E-Mail: hooshang_mirzaie@yahoo.com 


\section{Extended Abstract}

\section{Introduction}

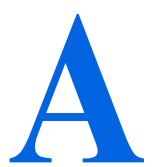

utism Spectrum Disorder (ASD) is neurodevelopmental syndrome with diverse phenotypes. Its main features are high social communication deficit and repetitive and restrictive behaviors [1]. Studies have reported that the prevalence of this disorder is increasing [2]. It affects all areas, especially social participation, sleeping, daily living activities, education and playing [2]. Children with ASD can play, although the game of their choice or its quality and duration may seem unusual to healthy children. Play-based therapeutic intervention is recommended for these children [2,3]. Play is the best way to help a child with ASD. Parents of these children can approach their children through play $[4,5]$. Studies show that mothers of children with ASD experience more stress than mothers with normal children [6]. It should be noted that it is not only the upbringing of a ASD child that creates a psychological burden for the family, but also the inability to establish mutual interactions, the child's understanding, and communication between family members, which makes cause the family to face problems [7].

Mother's mood affects the behavioral problems of ASD children and even their fathers' mental health, and this can facilitate or disrupt the recovery process of these children $[8,9]$. Therefore, it seems necessary to find an effective way to reduce the stress of mothers of ASD children and thus increase the child's performance. In Iran, methods such as positive parenting program, parenting skills program, parental thinking program, resilience training program, and response-based methods have been used to investigate the effect of stress in mothers of these children [10-14]. These methods only examined the mothers and did not involve the children in the intervention. The best intervention method that can involve both mother and child is play therapy [1, 15]. Filial therapy is a family-centered play therapy that uses play as a primary tool to help parents and children communicate, work on their problems, and build a stronger relationship [16]. It was introduced by Bernard and Louise Guerney [23].

The ten-session filial therapy model developed by Gary Landreth [17] is one of the most widely used treatment protocols in this approach which is called Child Parent Relationship Therapy (CPRT). In this study, we used this protocol. Although there is evidence of high levels of stress in mothers of children with ASD, studies that have conducted in the field of ASD have focused more on the problems of ASD children and few interventions have been proposed to reduce maternal stress [18]. The aim of this study was to investigate the effect of filial therapy on reducing stress of mothers of children with ASD.

\section{Materials \& Methods}

This is an experimental study with Pre-test/Post-test design using control group. The study population consists of all mothers of children with ASD aged 4-12 years referred to the occupational therapy and autism centers in Isfahan, Iran. First, the research objectives and method were explained to all mothers. Then, the mothers who volunteered to participate in the intervention signed a consent form, and then completed a demographic form, Gilliam Autism Rating Scale-2 (GARS-2), and Abidin's Parenting Stress Index-Short Form (PSI-SF). Then they were randomly divided into two groups of intervention and control. The sample size was calculated 40 according to the Formula 1. Since filial therapy groups consist of 8 people, the sample size was determined 32 . There were 4 groups of 8 assigned into two groups of intervention and control.

1. $2 N=\frac{4\left(Z_{\alpha}+Z_{\beta}\right)^{2} \sigma^{2}}{\delta^{2}}$

The PSI-SF has 36 items developed by Abidin [19]. It has three subscales of Parental Distress (PD), ParentChild Dysfunctional Interaction (PCDI), and Difficult Child (DC). This questionnaire has acceptable reliability and validity to measure maternal stress [20]. The GARS-2 is a checklist designed by Gilliam (1994) to diagnose autism in the age group of 3-22 years. It has acceptable reliability and validity to measure autism. We used this tool to match children in the intervention and control groups.

Mothers participated in filial therapy sessions in groups of 8, each for 1-2 hours, once a week. After each session, there was a 30-min play session with the children; during this time, they followed what they had learned in the therapy sessions while playing with their child. Then, at the beginning of the next session, the therapist and the mothers reviewed the previous topics, answered the questions, reviewed the parenting booklets that should be completed for each session, and watched the parent-child play videos. At the end of the 10-week intervention period, the PSI-SF was completed again by the mothers in both groups. The mean scores of PSISF and its subscales before and after the intervention in each group were compared by using paired t-test and independent t-test. ANCOVA and Eta squared coefficient 
were used to determine the effect of intervention on the PSI-SF score. Data analysis was performed in SPSS by considering a significance level of $\mathrm{P}<0.05$.

\section{Results}

Participants included 9 girls ( 5 in the intervention group and 4 in the control group) and 23 boys (11 in the intervention group and 12 in the control group). The subjects in the two groups were not significantly different in terms of mother's occupation, mother's age, number of children, child's educational level, child's GARS score, child's gender and child's age (Table 1). Shapiro-Wilk test was used to evaluate the normality of overall parenting stress data distribution in the Pre-test and Post-test phases. As shown in Table 2, the results of paired t-test showed no statisti- cally significant difference between the two groups at baseline $(\mathrm{P}=0.679)$ but a significant difference in the Post-test phase $(\mathrm{P}=0.010)$. The results of independent t-test showed that the difference in overall PSI-SF scores between Pretest and Post-test phases in the control group was not significant $(\mathrm{P}=0.268)$ but there was a significant difference in the intervention group $(\mathrm{P}=0.001)$. Findings indicate that filial therapy caused significant changes in the intervention group compared to the control group.

The result of ANCOVA presented in Table 3 showed that the Pre-test score of parenting stress had an effect on the overall parenting stress after intervention, such that it could explain $82 \%$ of the total parenting stress variation. After controlling the Pre-test score, the mean overall parenting stress score became significantly different between

Table 1. Mean and standard deviation of mother's and child's age and GARS score in two study groups

\begin{tabular}{ccccccc}
\hline & \multicolumn{4}{c}{ Mean \pm SD } & \multicolumn{2}{c}{ t-test Results } \\
\cline { 2 - 7 } Characteristics & \multicolumn{2}{c}{ Intervention Group } & \multicolumn{2}{c}{ Control Group } & t & P \\
\cline { 2 - 7 } & 7.56 & 1.45 & 7.50 & 1.36 & 0.125 & 0.901 \\
child's age & 34 & 4.13 & 35.93 & 5.72 & -1.098 & 0.281 \\
mother's age & 57.81 & 10.25 & 58.50 & 9.80 & -0.194 & 0.848 \\
\hline child's GARS score & & & & & & \\
\hline
\end{tabular}

Table 2. Comparing the overall parenting stress of mothers in two study groups

\begin{tabular}{|c|c|c|c|c|c|c|c|}
\hline \multirow{2}{*}{ Variable } & \multirow{2}{*}{ Phase } & \multicolumn{4}{|c|}{ Mean $\pm S D$} & \multirow{2}{*}{$t$} & \multirow{2}{*}{$\mathbf{P}$} \\
\hline & & \multicolumn{2}{|c|}{ Intervention Group } & \multicolumn{2}{|c|}{ Control Group } & & \\
\hline \multirow{2}{*}{ Overall parenting stress } & Pre-test & 106.62 & 15.48 & 110.06 & 29.048 & -0.418 & 0.679 \\
\hline & Post-test & 86.50 & 9.72 & 108 & 28.32 & -2.872 & 0.010 \\
\hline Paired t-test & \multicolumn{3}{|c|}{6.781} & \multicolumn{2}{|c|}{1.149} & & \\
\hline $\mathrm{P}$ & \multicolumn{3}{|c|}{0.001} & \multicolumn{2}{|c|}{0.268} & & \\
\hline
\end{tabular}

Rehabilitation

Table 3. ANCOVA results of the effect of filial therapy on overall parenting stress of mothers

\begin{tabular}{rcccccc}
\hline Source & Sum of Squares & df & Mean Square & F & P & Eta Squared \\
\hline Group & 277.566 & 1 & 277.566 & 33.625 & 0.001 & 0.537 \\
\hline Pre-test & 11.64 .515 & 1 & 11.64 .515 & 134.285 & 0.001 & 0.822 \\
\hline Error & 2389.485 & 29 & 82.396 & & & \\
\hline
\end{tabular}


the two groups $(\mathrm{P}=0.001)$. According to Eta squared value, the group filial therapy can explain $54 \%$ of the changes in the total parenting stress of mothers. Overall, it can be said that group filial therapy can reduce the parenting stress of mothers.

\section{Discussion}

The purpose of this study was to investigate the effect of filial therapy on parenting stress in mothers of children with ASD. Statistical findings showed that the mean difference in parenting stress between the two groups after the intervention was significantly different, and indicated that filial therapy can be effective in reducing their parenting stress. Few studies have been conducted in Iran using filial therapy; however, the interest in research in this field is increasing. For example, Mirzaei [22] compared the effects of filial therapy and therapist-child play therapy on the parenting stress of the mothers of children with attention deficit hyperactivity disorder. Both methods reduced the stress of mothers, but filial therapy was more effective. Although the study population was different, his results are consistent with our findings. In another study, Nejati et al. [23] investigated the effectiveness of CPRT on improving maternal parenting styles in hyperactive children. Samples were 30 mothers divided randomly into two groups of intervention and control. Findings showed that the mothers in the intervention group had a significant increase in using authoritative parenting style compared to the control group. Their study revealed that filial therapy has a positive effect on parenting styles of mothers.

Soltani [24] examined the effect of child-parent play therapy on 20 mothers of children with autism in Mashhad. Play therapy increased mothers' self-efficacy and selfconfidence. In his study, mothers' training sessions were conducted individually with their children. Play therapy should be conducted in group; however, positive results were reported which is consistent with our findings. Moqavam [25] studied the effectiveness of CPRT on the stress, anxiety, and depression of 30 mothers with a mild mental disability child. The results showed that filial therapy reduced stress in mothers which is consistent with our findings. Becklof [26] evaluated the effect of filial therapy on children with pervasive development disorder. Twenty-two parents of children aged 3-10 years with this disorder were divided into control and intervention groups. The intervention group received 10 CPRT training sessions (one 2-hour session per week) and 7 play therapy sessions with their children (one 30-minute session per week). The results showed that the intervention 1 group was significantly improved in accepting their child's need for autonomy and in- dependence as well as their child's overall acceptance. This is in agreement with the results of present study.

\section{Conclusion}

Autism is a disorder with a complex nature and various signs and symptoms that sometimes it even makes it difficult for psychiatrists and specialists to diagnose. Therefore, finding the best and most effective treatment for these children is difficult and its costs are high. On the other hand, due to the special characteristics of these children (stereotyped movements, not starting to communicate with others or continuing it, sensory problems, etc.), family members and especially mothers due to closer proximity to the child, face with many problems such as: stress, depression, increased costs, lack of social understanding, no participation in social activities, and spending a lot of time educating the child. Most mothers think that their children and their development are the first priority and sometimes they forget about themselves and their mental and even physical health. This has a negative effect on the growth and treatment of children with autism. With a focus on CPRT, filial therapy is a technique without limitations that in a time process can move both mother and child on the path to treatment. It can reduce the burden of stress and unhappiness of mothers by creating more acceptance of the child and positively affect the developmental and therapeutic process of children, directly and indirectly. It has no side effects and all people with different cultures can benefit from this treatment protocol.

The present study, like any other studies, had inevitable limitations. For example, since the questionnaires were completed by parents, they may have evaluated the symptoms better or worse than the actual situation. Moreover, due to the label of autism, many mothers were afraid to participate in the group therapy and introduce their child, and were reluctant to express their real opinions. Further studies are suggested on the mothers of other exceptional children or examining the effect of filial therapy on the stress of the fathers of ASD children. There was no follow-up assessment in the present study. More studies with a follow-up are recommended to determine the time effect on the results of the intervention. The present study was related to mothers in Isfahan; further studies can be conducted in other cities of Iran.

\section{Ethical Considerations}

\section{Compliance with ethical guidelines}

This study was approved by the Ethics Committee of University of Social Welfare and Rehabilitation Sciences (Code: IR.USWR.REC.1396.97). 
Funding

The present paper was extracted from the MSc. thesis of the first author, Department of Occupational Therapy, School of Rehabilitation, University of Social Welfare and Rehabilitation Sciences.

\section{Authors' contributions}

Ideation and conceptualization: Houshang Mirzaei and Zahra Kiani; Research analysis: All authors; Review of sources: Hossein Sortiji, Zahra Kiani, Elahe Ebrahimi; Drafting, editing and finalization: Houshang Mirzaei, Hossein Sortiji, Zahra Kiani; Supervision: Houshang Mirzaei, Hossein Sortiji, Samaneh Hosseinzadeh; Management: Houshang Mirzaei.

\section{Conflicts of interest}

The authors declared no conflict of interest.

\section{Acknowledgements}

The authors would like to thank the staffs of Mani and Borna occupational therapy clinics as well as the autism centers Yavaran Zainab and Ordibehesht. 


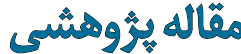 \\ بررسى ثأثير فيليال ترايى بر تنش كل فرزنديرورى مادران كودكان أتيسم
}

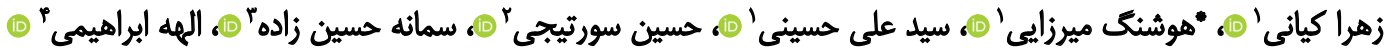

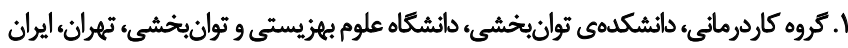

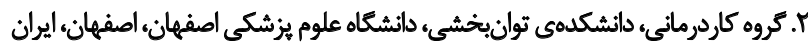

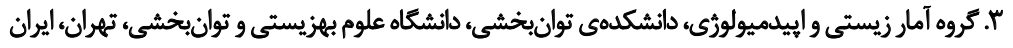

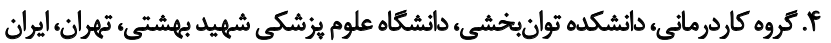

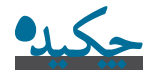

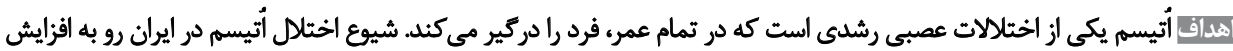

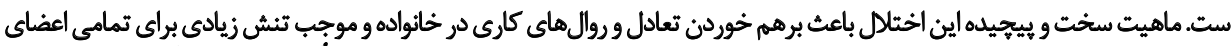

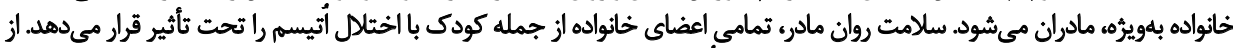

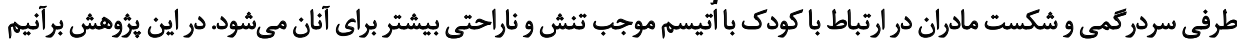

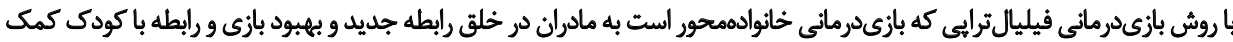

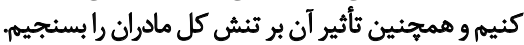

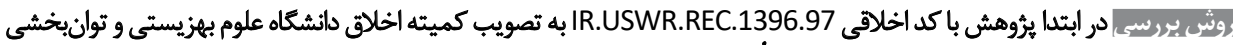

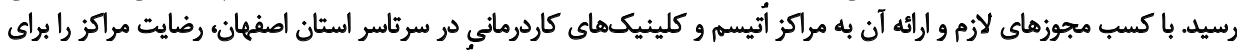

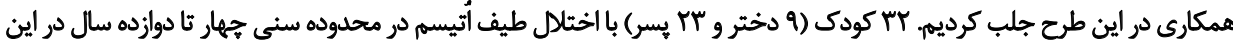

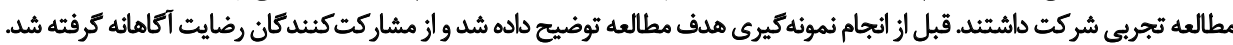

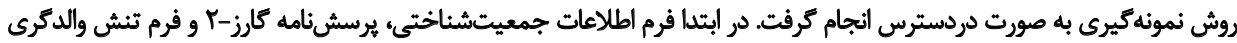

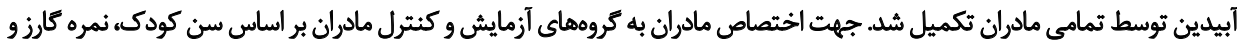

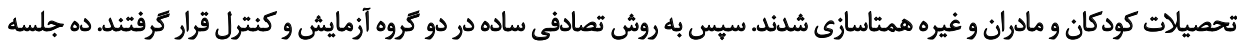

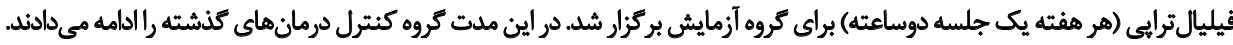

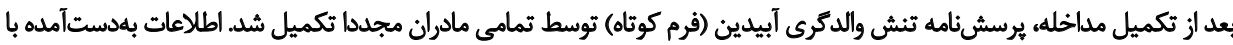

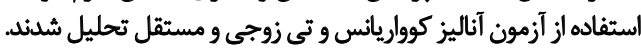

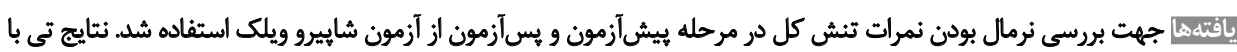

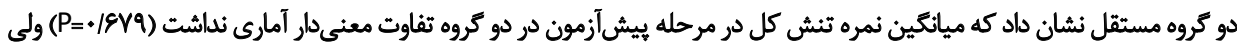

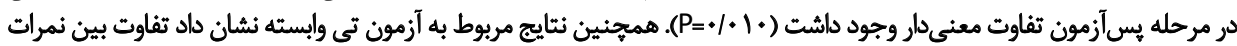

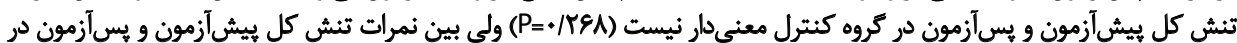

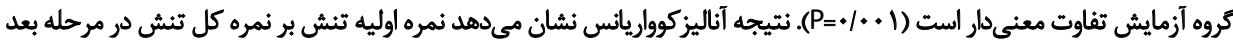

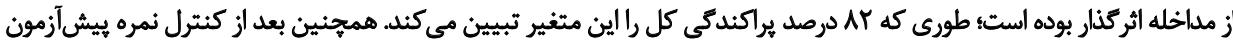

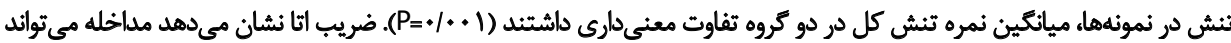

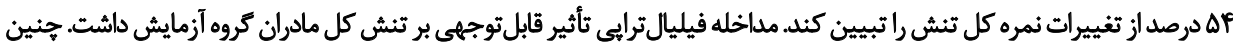

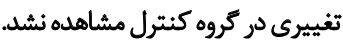

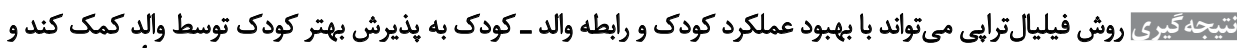

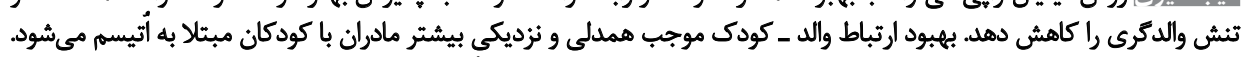

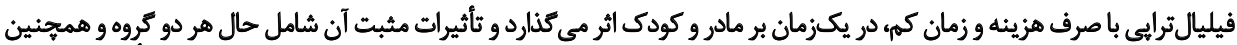

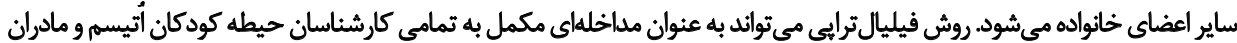
أنان آموزش داده شود.

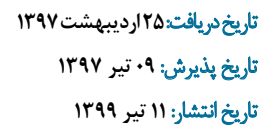


خلق مادر بر مشكلات رفتارى كودكان مبتلا به أتيسم و حتى بـتي

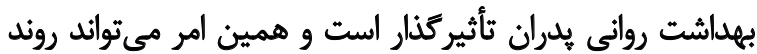

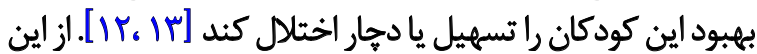

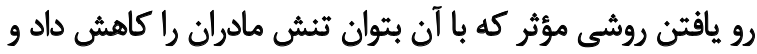

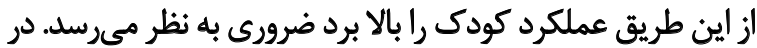

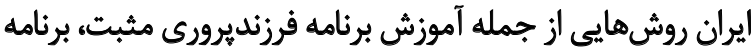

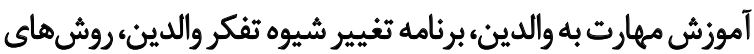

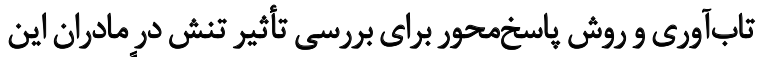

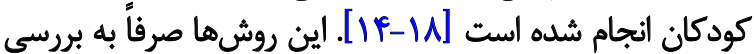

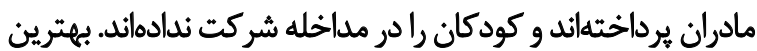

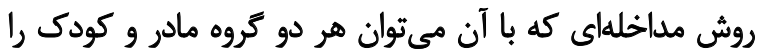

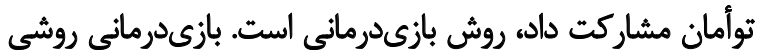

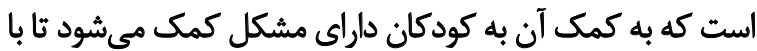

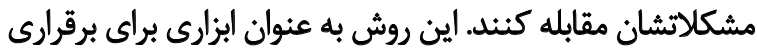

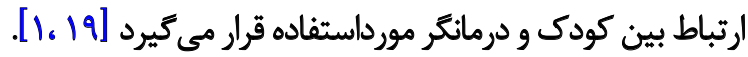

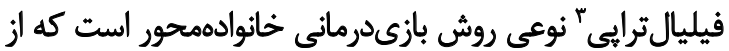

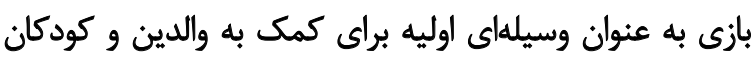

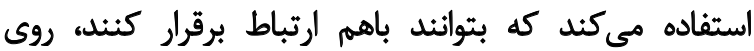

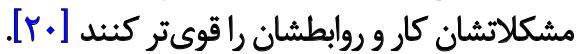
امروزه فيليالترايى رويكردى شناختهشده است و مكرراً توسط داني

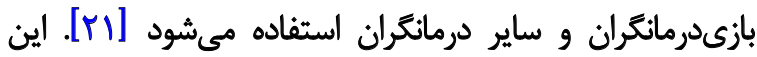

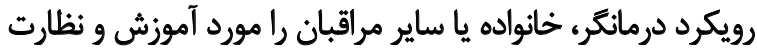

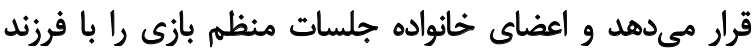

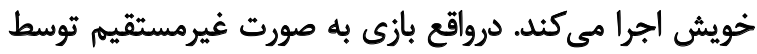

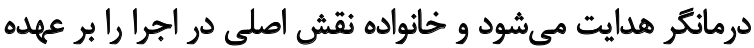

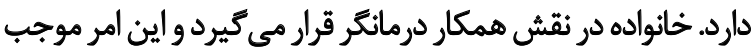

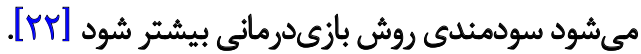

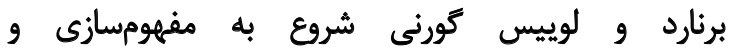

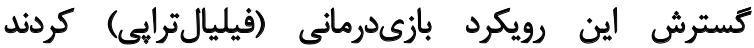

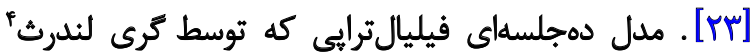

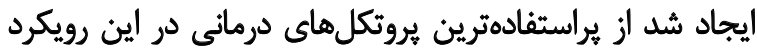

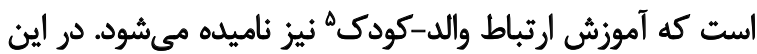

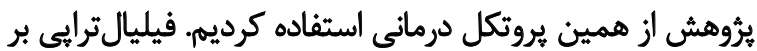

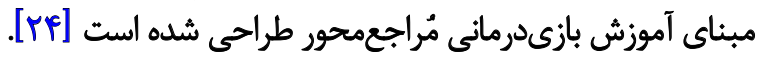

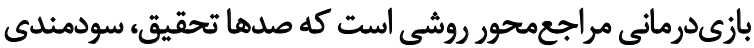

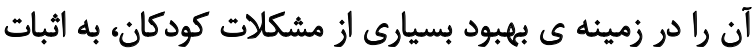

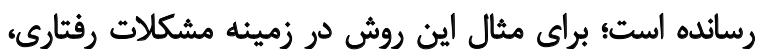

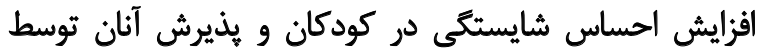

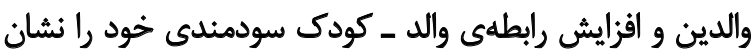

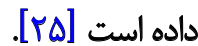

3. Filial therapy

4. Gary Landerth

5. Child parent relation therapy
مقدمه

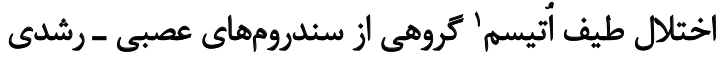

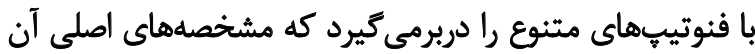

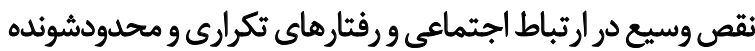

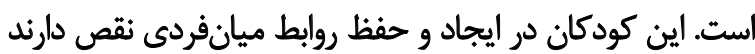

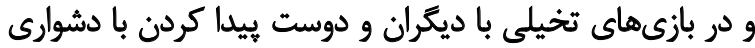

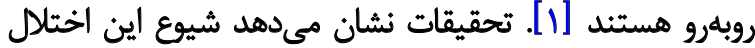

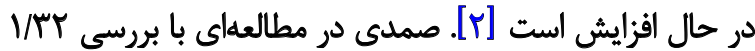

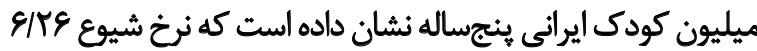

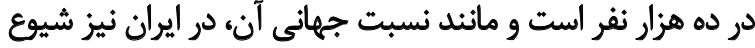

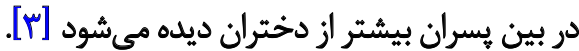

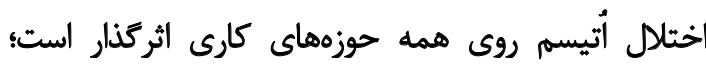

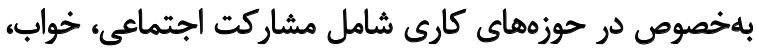

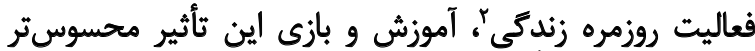

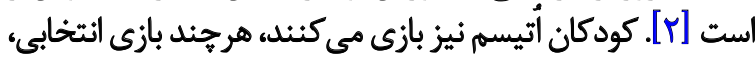

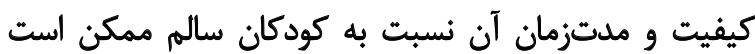

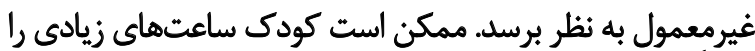

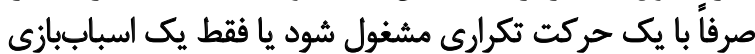

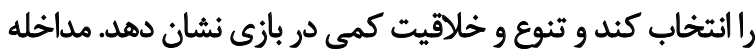

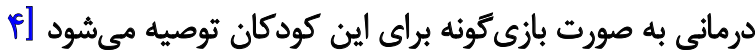

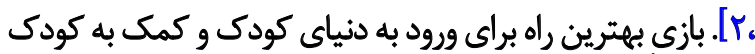

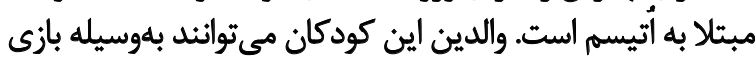

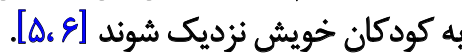
با توجه به طيف كسترده و متنوع أتيسم، هر كودكي نقاط

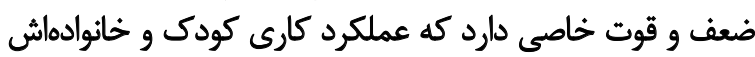

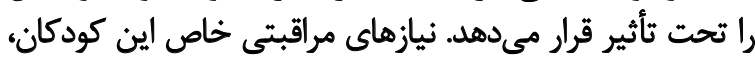

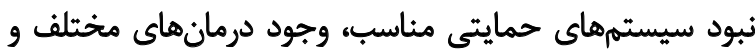

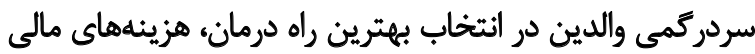

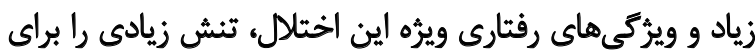

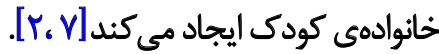
بررسى بها نشان ميدهند مادران كودكان مبتلا به أتيسم تنش بودي

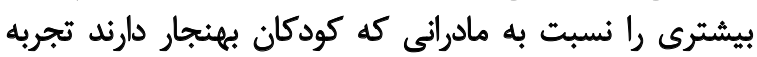

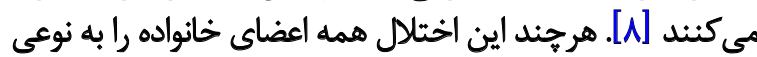

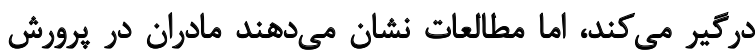

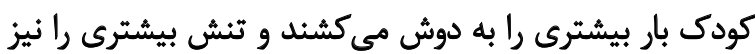

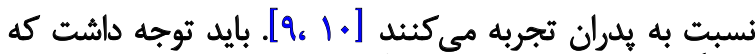

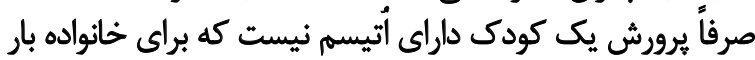

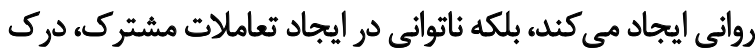

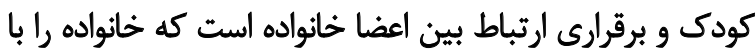

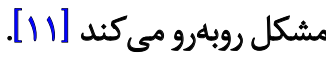

1. Autism sepectrum disorder

2. Activities of daily living 
أتيسم توسط روانيزشك متخصص و ثبت شدن اختلال أتيسم

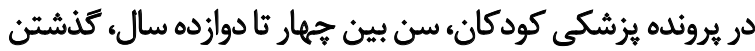

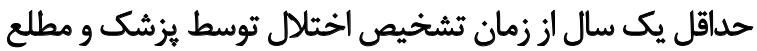

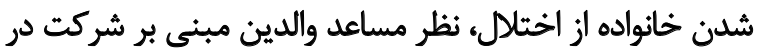

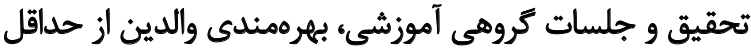

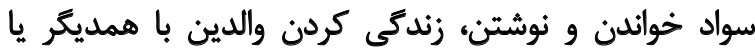

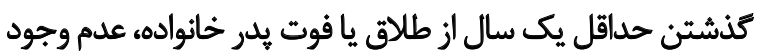

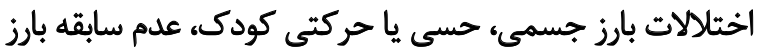

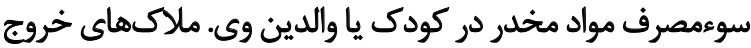

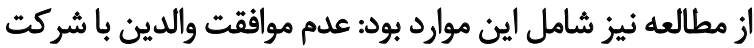

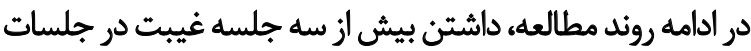

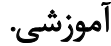

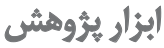

در اين يُروهش از دو آزمون استفاده كرديد:

\section{شاخص تنش والدين (فرم كوتاه)'}

اين مقياس نسخه كوتاه عاسؤالى از شاخص اصلى تنش

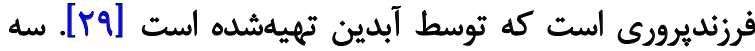

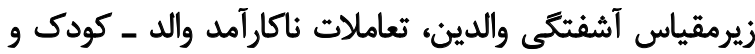

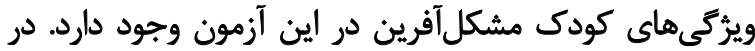

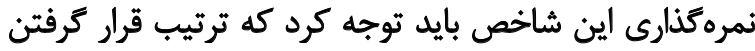

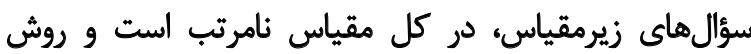

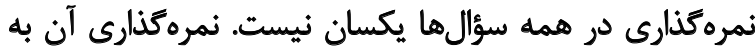

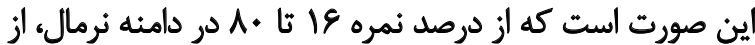

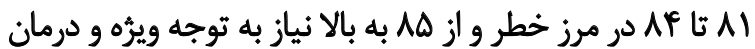

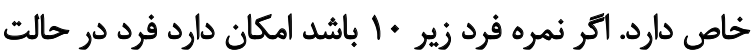

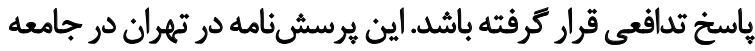

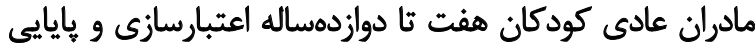

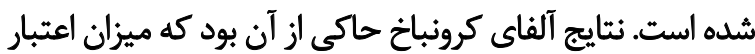

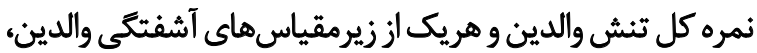

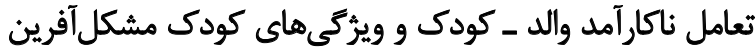

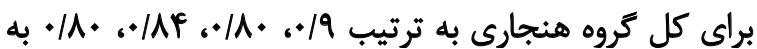

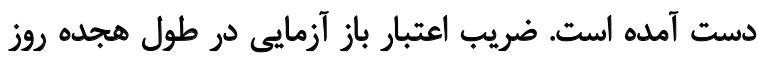

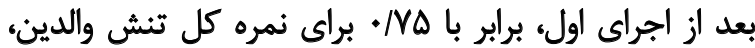

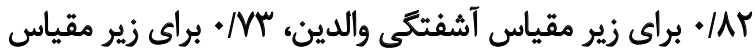

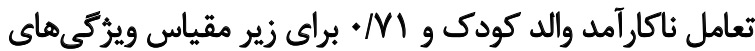

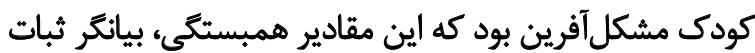

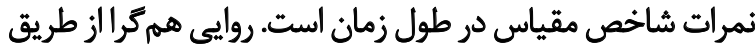

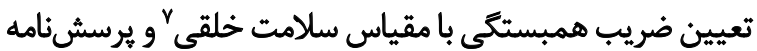

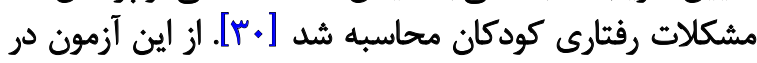

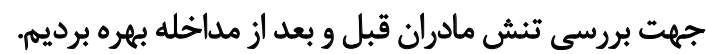

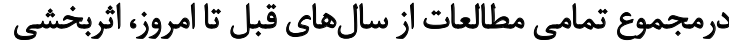

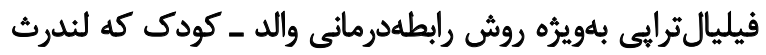

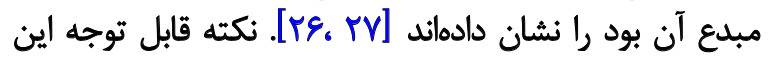

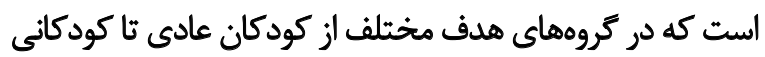

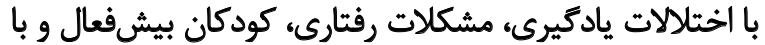

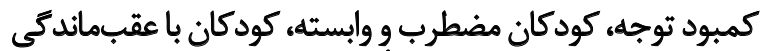

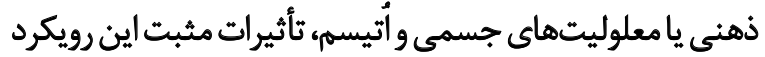

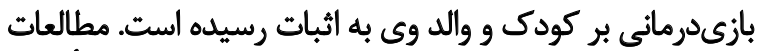

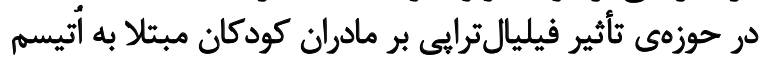

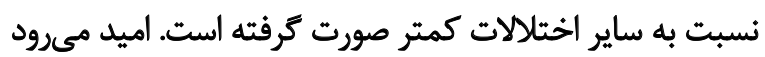

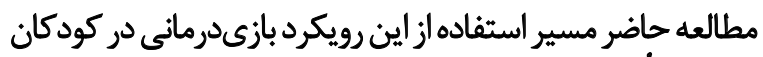

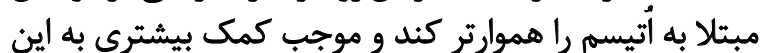

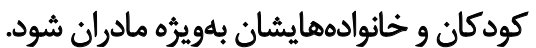

با وجودى كه شواهد حاكى از تنش زياد در مادران كودكان

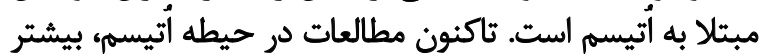

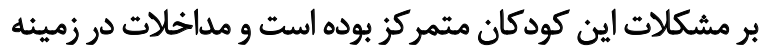

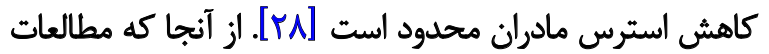

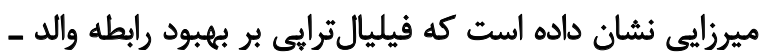

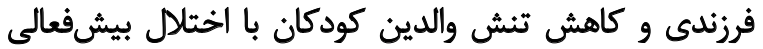

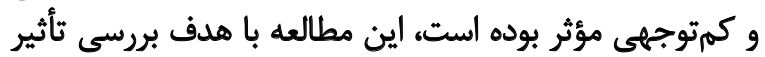

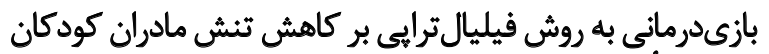
مبتلا به أتيسم انجام شد بهد

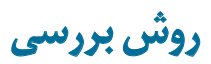

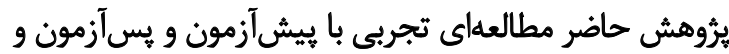

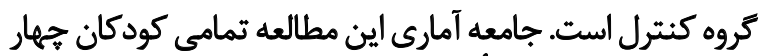

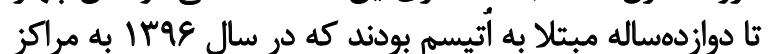

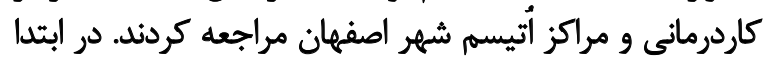

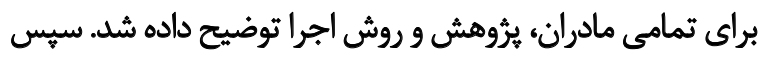

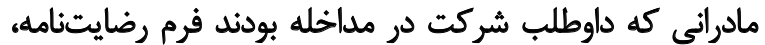

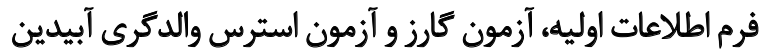

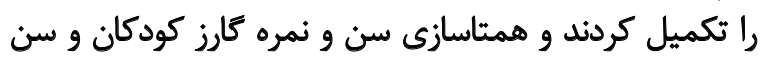

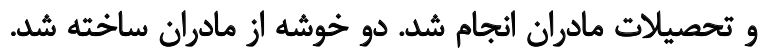

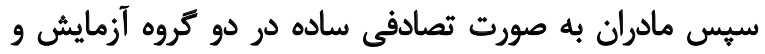

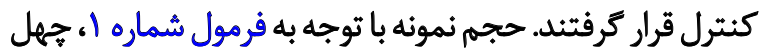

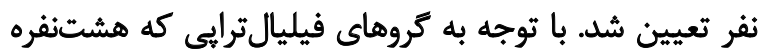

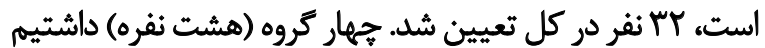

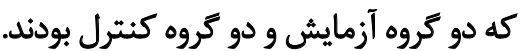

$$
2 N=\frac{4\left(Z_{\alpha}+Z_{\beta}\right)^{2} \sigma^{2}}{\delta^{2}}
$$

ملاكهاى ورود به مطالعه عبارت بود از: تشخيص اختلال 


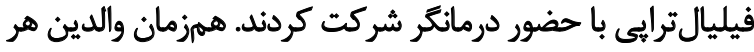

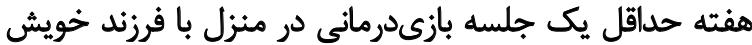

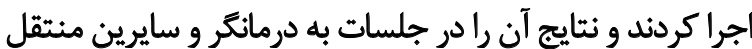

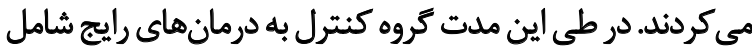

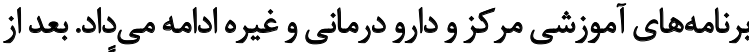

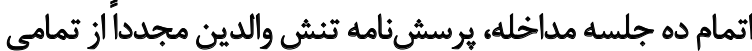
مادران گرفته شد و نتايج كزارش شد.

ساختّار مداخله

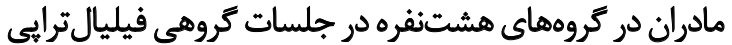

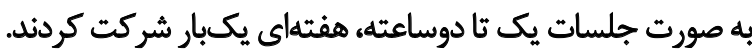

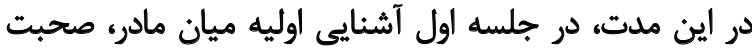
در مورد كودى و احساسات خودشان و آشنايى كلى بإئ با اختلال

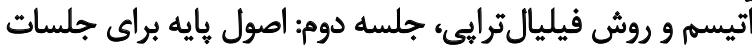

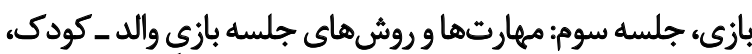

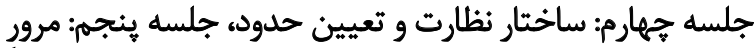

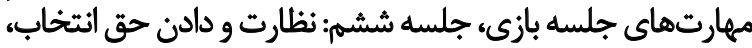

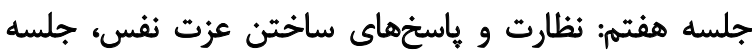

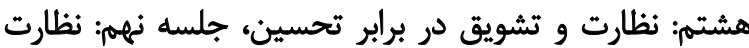

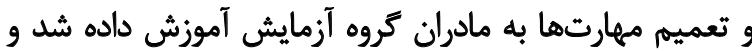

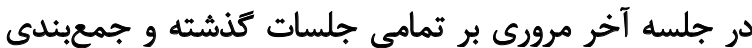

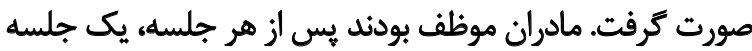

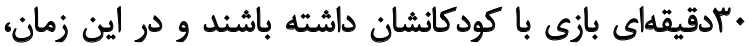

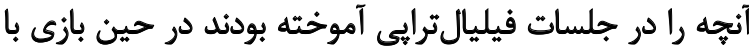

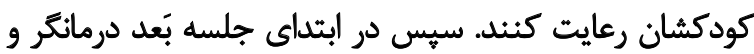

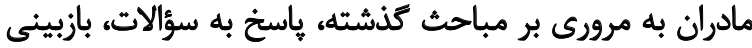

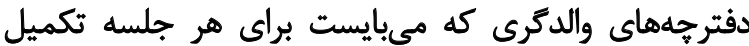

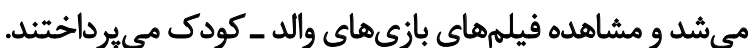

\section{روش تجزيه و تحليل أمارى}

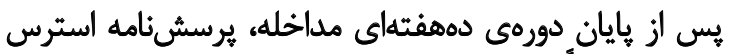

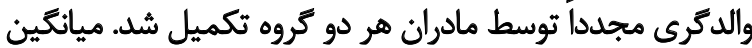

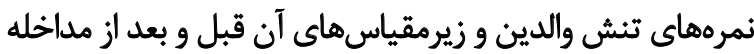

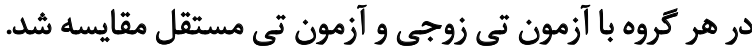

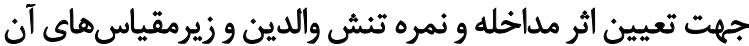

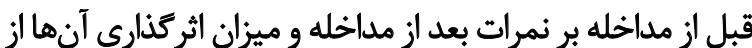

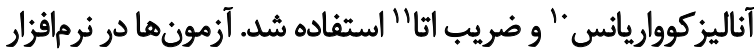

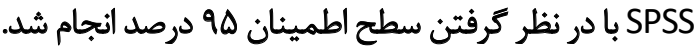

يافتهها آزمودنىها شامل 9 دختر (ه نفر در كروه آزمايش و F نفر در

\section{آزمون تشخيصى أُتيسم كيليام (كارز-Y)}

آزمون گارز جك ليستى است كه توسط كيليام" براى تشخيص

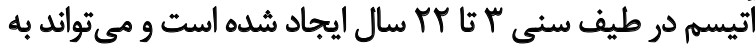

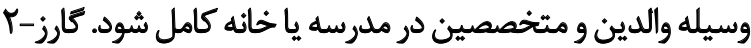

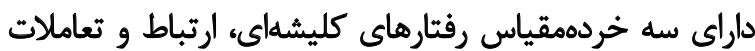

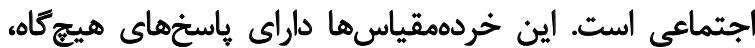

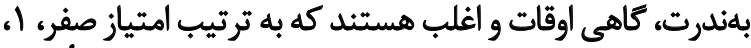

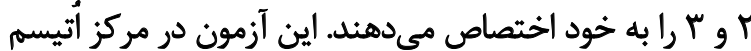

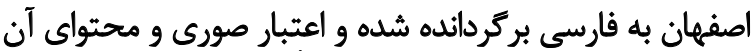

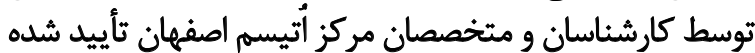

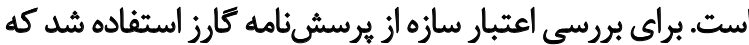

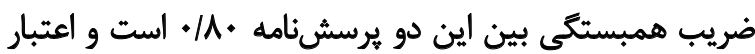

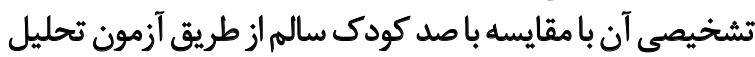

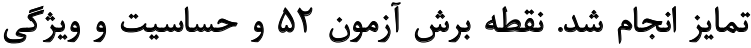

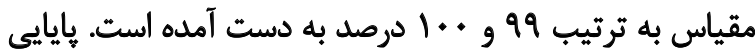

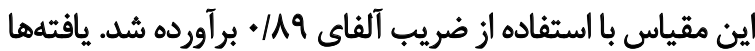

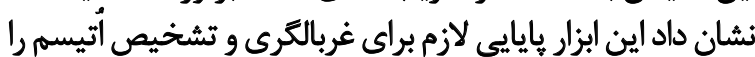

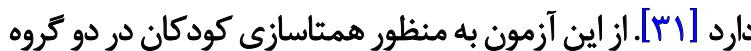
آزمايش و كنترل بهره برديم. (ارن.

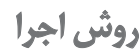

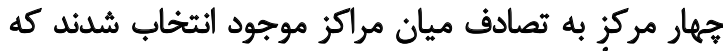

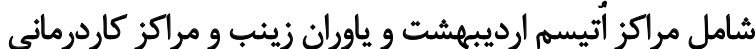
مانى و برنا بودند.

سيس طبق ملاكهاى ورود، يروندههاى كودكان را بررسى

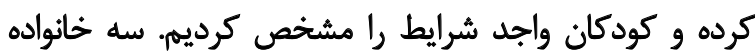

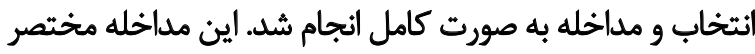

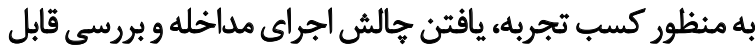

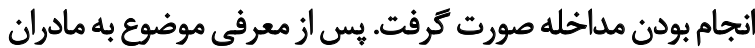

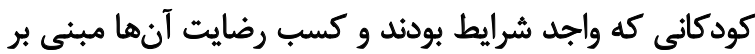

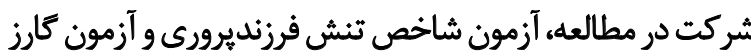

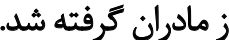

سيس ميان مادران سعى شد بيشترين همتاسازى شامل سن

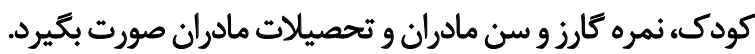

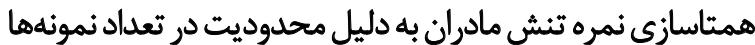

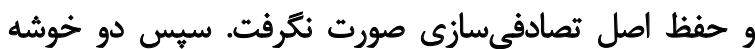

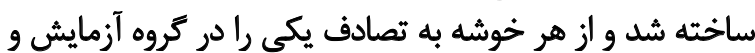

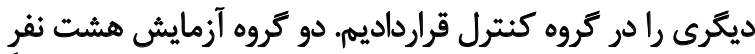

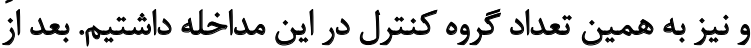
آن مادران در ده جلسه آموزشى (هر هفته يك جلسه دون دوساعته) 
نمره كل تنش را تبيين كند. با توجه به جدول شماره بّ، مداخله فيليال ترايى موجب كاهش تنش والد تبرى كن مادران شده است.

بحث

ماثروهش حاضر با هدف بررسى تأثير فيليالترايى بر تنش بأنش

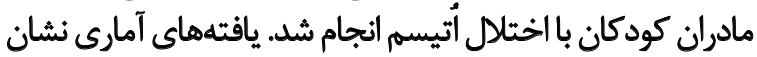

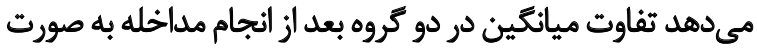

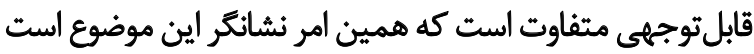

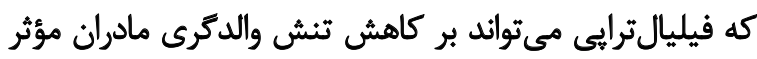

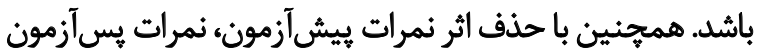

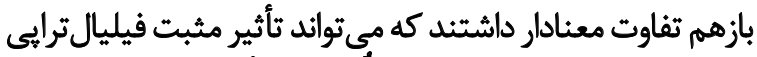
بر تنش مادران كودكان مبتلا به أثيسم را تأييد كندائد

يافتههاى مطالعه حاضر نشان داد بازيدرمانى به به روش

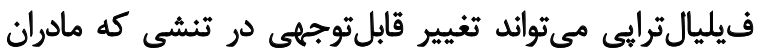

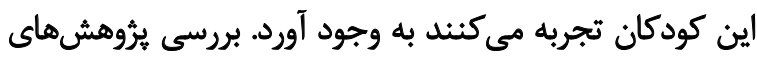

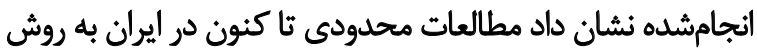

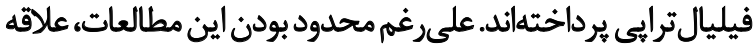

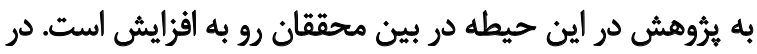

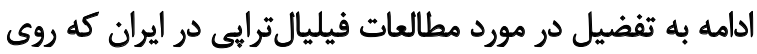
ساير كروههاى هدف صورت كرفته است توضيح خواهيم داد.

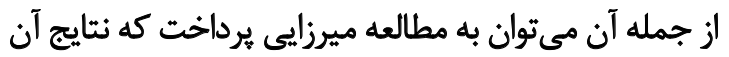

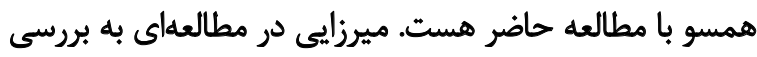

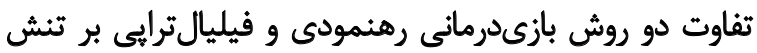

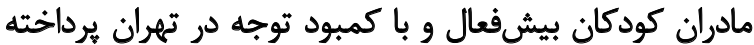

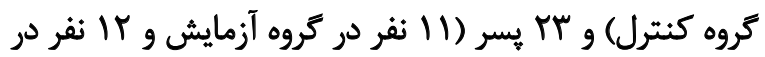

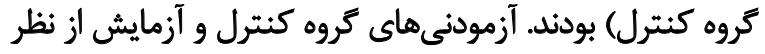

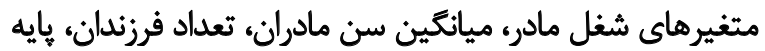

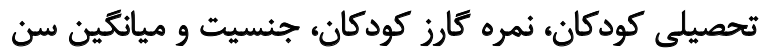
كودكان تفاوت معنادارى نداشتند (جدول شمار كمان، جناره (1). جهت بررسى نرمال بودن نمرات تنش كل در مر مرحله

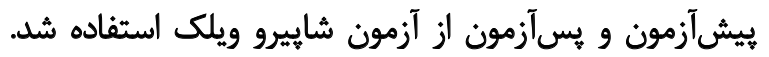

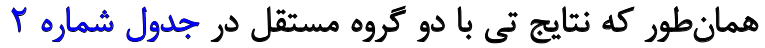

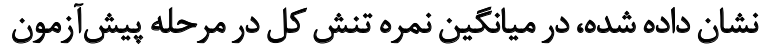

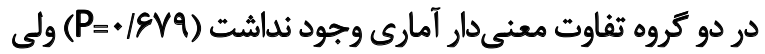

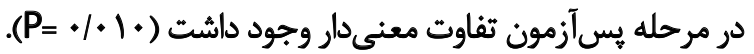
همجِين نتايج مربوط به آزمون تى وابسته نشان ميدهد

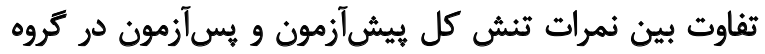

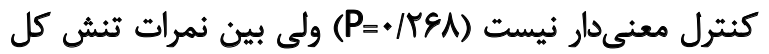

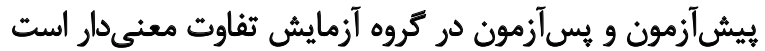

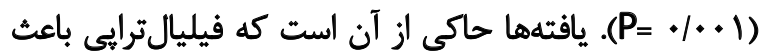

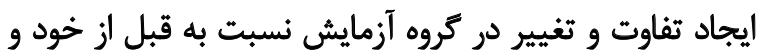
همجنين نسبت به كروه كنترل شده است

نتيجه آناليز كوواريانس نشان مىدهد نمره اوليه تنش بر نمره

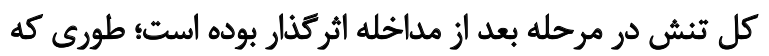

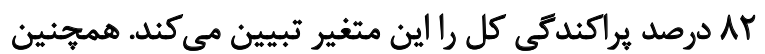

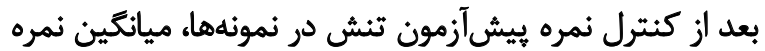

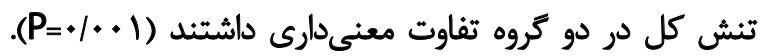

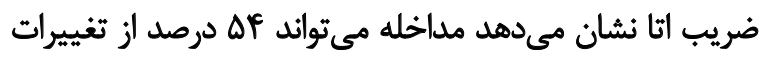

جدول ا. ميانكين و انحراف معيار سن كودك، سن مادر و نمره كارز در مروههاى آزمايش و كثترل

\begin{tabular}{|c|c|c|c|c|}
\hline \multicolumn{2}{|c|}{ آزمون تى دو كروه مستقل } & \multicolumn{2}{|c|}{ ميانكين士 انحراف معيار } & \multirow{2}{*}{ شن كودى أمارى } \\
\hline $\mathbf{P}$ & $\mathrm{t}$ & كتترل & أزمايش & \\
\hline$+/ 9+1$ & $\cdot / 1 T \Delta$ & $V / \Delta+ \pm 1 / F \varepsilon$ & $V / \Delta \& \pm V / \uparrow \Delta$ & سن كودك \\
\hline$\cdot /$ / Al & $-1 / \cdot 4$ & $r \Delta / q \Psi \pm \Delta / N$ & 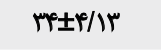 & سن مادر مار \\
\hline$\cdot / A F \Lambda$ &.$- / 199$ & $\Delta N \Delta \cdot \pm q / \Lambda$. & $\Delta V / A) \pm 1 . / T \Delta$ & نمره كارز \\
\hline
\end{tabular}

جدول Y. مقايسه ميانكين نمره تنش كل مادران داراي كودكان أتيسم در كروههاي موردمطالعه

\begin{tabular}{|c|c|c|c|c|c|}
\hline \multirow{2}{*}{$\mathbf{P}$} & \multirow{2}{*}{$\mathbf{t}$} & \multicolumn{2}{|c|}{ ميانكين土اثحراف معيار } & \multirow{2}{*}{ مرحله } & \multirow{2}{*}{ متغير } \\
\hline & & كنترل & أزمايش & & \\
\hline $.18 v 9$ & $-+/ F 11$ & $11+1 \cdot q \pm+r q++1$ & $1+\& / g / \pm \pm 1 \Delta / \% A$ & يميش آزمون & \\
\hline \multirow[t]{3}{*}{$.1+1$} & $-r / A R T$ & $1+\lambda \pm T N T T$ & $N / \Delta+ \pm q / N$ & يسآزمون & تش (نمره كل) \\
\hline & & MPq & ENAI & & تى زوجى \\
\hline & & - MEA & $.1 .+1$ & & $P$ \\
\hline
\end{tabular}


جدول "ب. نتيجه آناليزكوواريائس تنش كل مادران

\begin{tabular}{|c|c|c|c|c|c|c|}
\hline ضريب اتا & $\mathbf{P}$ & Fماره F F F & ميانكين مربعات & درجه أزادى & مجموع مريعات & منيع \\
\hline . Iarr & $* 1+.1$ & MT/gTA & TW/ASF & 1 & IW/AES & كروه - اه \\
\hline \multirow[t]{2}{*}{. /ATr } & $* /+1$ & ITT/TAS & $11.94 / \Delta 10$ & 1 & $11.9 \% / \Delta 10$ & نمره ييش آزهون \\
\hline & & & AT/M & rq & 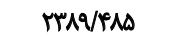 & خم \\
\hline
\end{tabular}

توانبخننى

در مطالعهاى بكلوف ثأثير فيليال ترايى بر كودكان طيف اختلالات

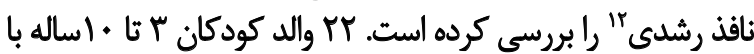

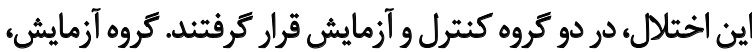

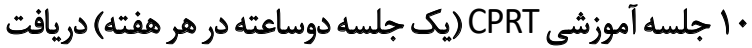

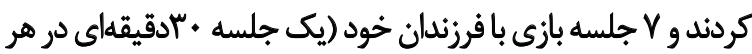

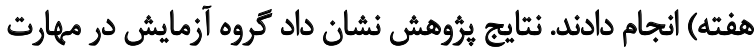
بذيرش نياز فرزندشان به خودمختارى و واستقلال، بهيبود جشمكيرى

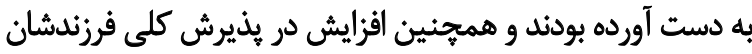

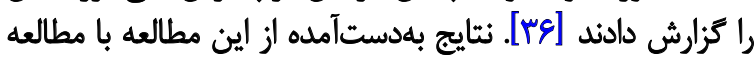

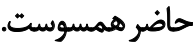
تنيجهيرى

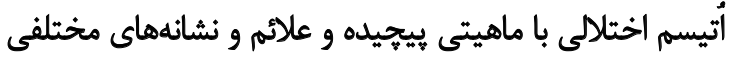

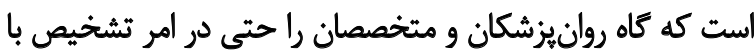

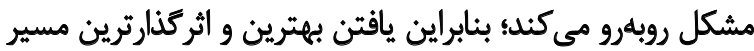

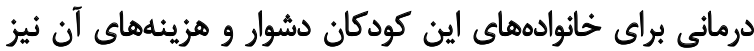

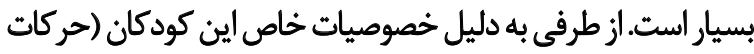

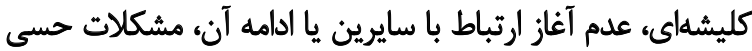

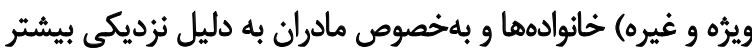

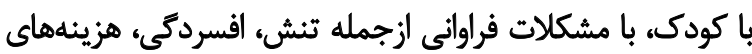

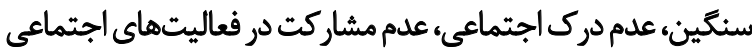

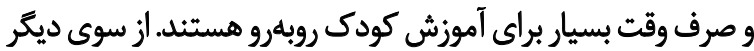

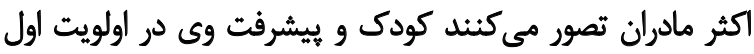

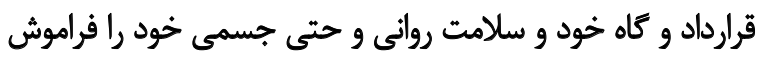

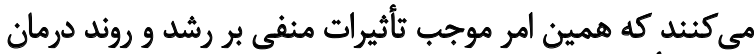

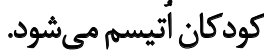

روش فيليالترائى با محوريت دادن به بازئردمانى كودكمهحور،

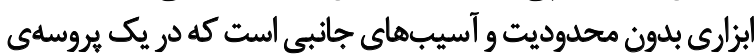

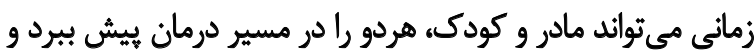

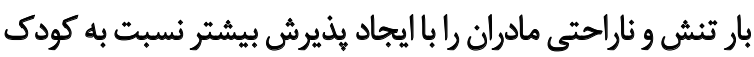

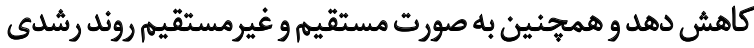

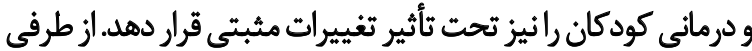

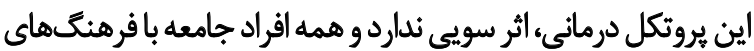
مختلف مى توانيد از اين يروتكل درمانى بهره برون ببرند.
است. نتايج مثايسه اين دو روش در آزمون تنش فرزنديرورى آبيدين

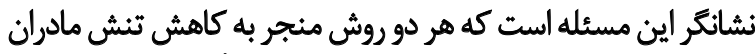

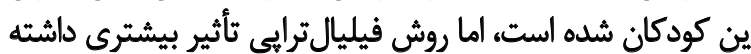

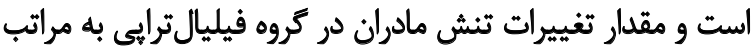

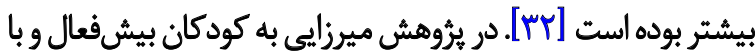

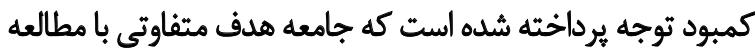

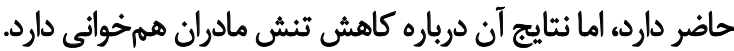

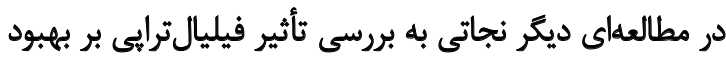

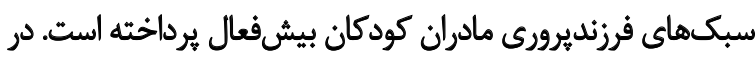

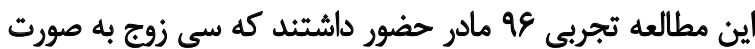

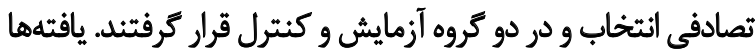

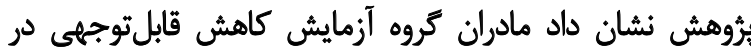

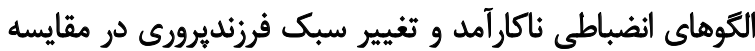

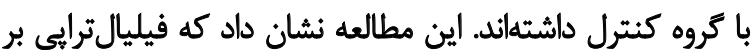

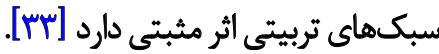

در مطالعهاى ديكر سلطانى به بررسى ثأثير آموزش همزئماني

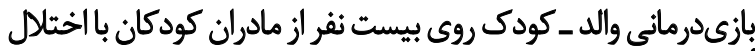

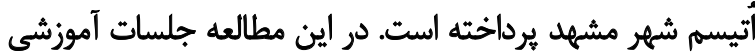

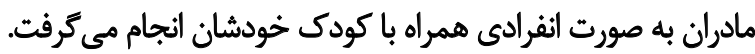

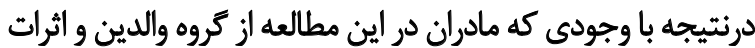

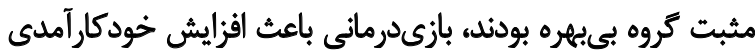

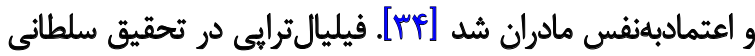

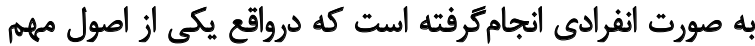

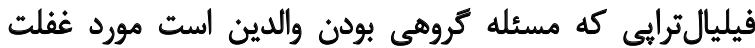

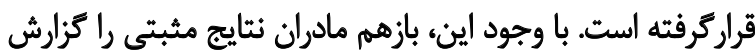
كردند كه با تحقيق حاضر همخ وخوانى دارد.

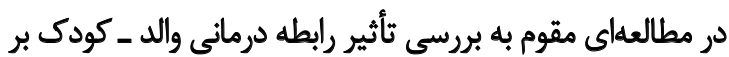

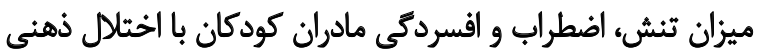

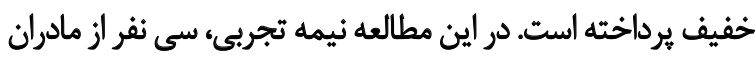

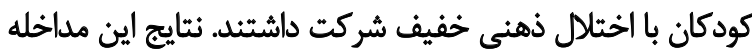

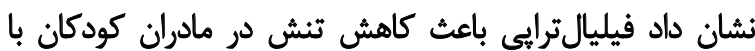

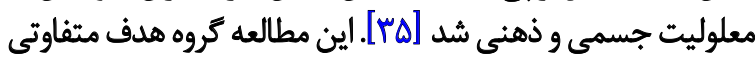

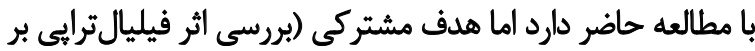
تنش مادران) را انبال مي كرده است. 


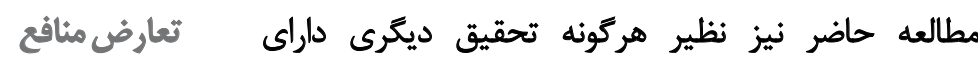

بنابر اظهار نويسندكان اين مقاله تعارض منافع ندارد.

$$
\text { تشكر و قدرواني }
$$

نويسندكان بر خود لازم مى إنداند از كارمندان كلينيكهاى

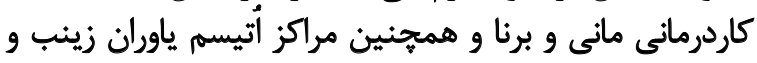
ارديبهشت مراتب تشكر و قدردانى خود راني ما ابراز كنند.

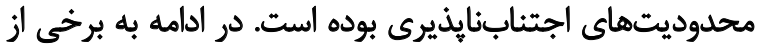

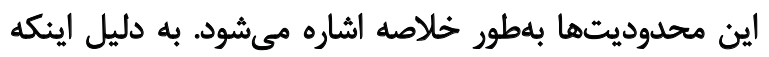

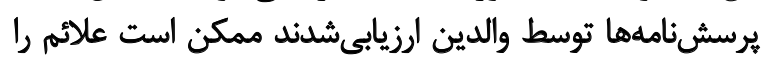

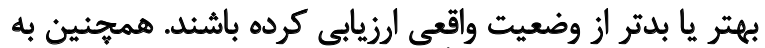

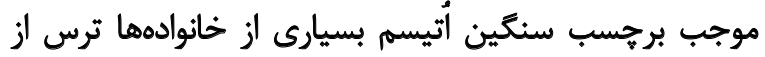

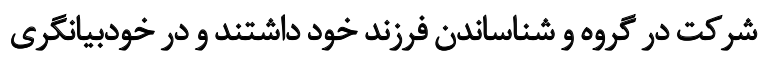
و اعلام نظرات واقعى امساك مى كردند.

اين يُوهش محدود به كودكان با اختلال أتيسم و مادران

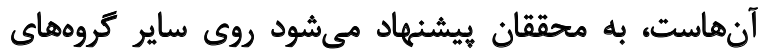

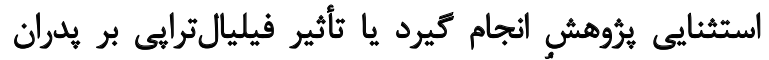

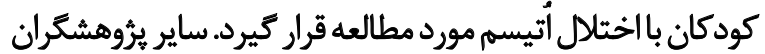

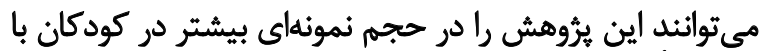

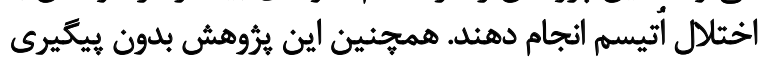

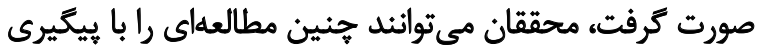

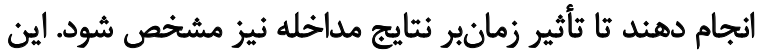

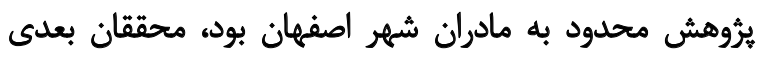
مى توانتد روى ساير شهرها نيز مداخله مشابه را انجام دهند.

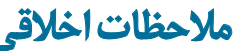

\section{ييروى أز اصول اخلاق يثؤهش}

يثروهش با كد اخلاقي IR.USWR.REC.1396.97 به تصويب

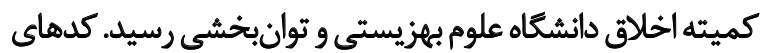

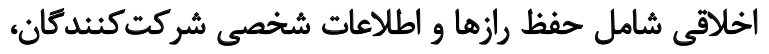

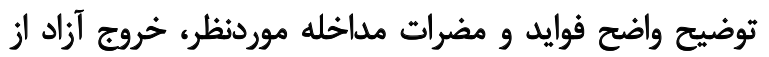

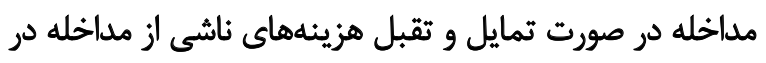
تمامى مراحل مطالعه مدنظر قرار كرفنند.

$$
\text { مامى مالى }
$$

اين مقاله از ياياننامه كارشناسى ارشد زهرا كيانى در كروه

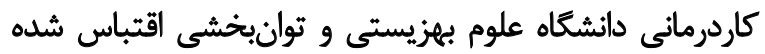

\section{مشاركت نويسئدكَان}

ايدهسازى و مفهومسازى: هوشنك ميرزايى و زهرا كيانى؛

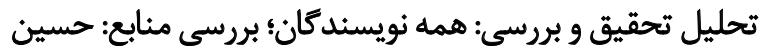

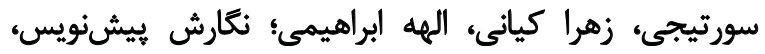

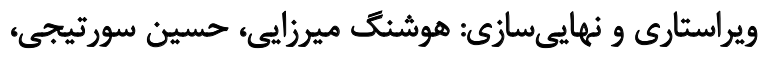

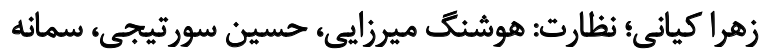
حسين زاده؛ مديريت: هوشنكَ ميرزايى. 


\section{References}

[1] American Psychiatric Association. Diagnostic and Statistical Manual of Mental Disorders $5^{\text {th }}$ ed. Washington DC: American Psychiatric Association; 2013.

[2] Case-Smith J, O’Brien JC. Occupational therapy for children and adolescents. Amsterdam: Elsevier Health Sciences; 2014.

[3] Samadi SA, Mahmoodizadeh A, McConkey R. A national study of the prevalence of autism among five-year-old children in Iran. Autism. 2012; 16(1):5-14. [DOI:10.1177/1362361311407091] [PMID]

[4] Charlop MH, Lang R, Rispoli M. All children can play: Prompting and modeling procedures to teach play to children with Autism Spectrum Disorder. Play and Social skills for children with autism spectrum disorder. New York: Springer; 2018. [DOI:10.1007/978-3-319-72500-0_3]

[5] Koegel RL, Schreibman L, Loos LM, Dirlich-Wilhelm H, Dunlap G, Robbins FR, et al. Consistent stress profiles in mothers of children with autism. Journal of Autism and Developmental Disorders. 1992; 22(2):205-16. [DOI:10.1007/BF01058151] [PMID]

[6] Jadidi Feighan M, Safrry S, Faramarzi S, Jamali Paghale S, Jadidi Feighan M. [Comparison of anxiety and social support of mothers of children with special needs and mothers with healthy children (Persian)]. Knowledge and Research in Applied Psychology. 2015; 16(2):43-52. http://jst-p.khuisf.ac.ir/article_533965_5776a 611287ef99eba7bf0e2f04533a0.pdf

[7] Keen D, Couzens D, Muspratt S, Rodger S. The effects of a parent-focused intervention for children with a recent diagnosis of autism spectrum disorder on parenting stress and competence. Research in Autism Spectrum Disorders. 2010; 4(2):229-41. [DOI:10.1016/j.rasd.2009.09.009]

[8] Khoramabadi R, Pouretemad HR, Tahmasian K, Chimeh N. A comparison of parenting stress in mothers of children with autistic disorder in mothers of children with normal. Family Research. 2013; 5(19):3879. https://www.sid.ir/fa/journal/ViewPaper.aspx?id=103709

[9] Afshari R. Study of coping strategies and mental health in mothers with autistic children. Tehran: University Of Social Welfare And Rehabilitation Sciences; 2004

[10] Behnia F, Rassafiani M, Nakhai S, Mohammadpour M, Ahmadi Kahjoogh M. [Time use of mothers of children with an Autism Spectrum Disorder: A comparative study (Persian)]. Iranian Rehabilitation Journal. 2017; 15(1):49-56. [DOI:10.18869/nrip.irj.15.1.49]

[11] Mirzaie H, Jamshidian E, Hosseini SA. [Routines in families of children with Autism (Persian)]. Archives of Rehabilitation. 2018; 19(3):184-93. [DOI:10.32598/ri.19.3.184]

[12] Hastings RP. Child behaviour problems and partner mental health as correlates of stress in mothers and fathers of children with autism. Journal of Intellectual Disability Research. 2003; 47(4-5):231-7. [DOI:10.1046/j.1365-2788.2003.00485.x] [PMID]

[13] Davis NO, Carter AS. Parenting stress in mothers and fathers of toddlers with Autism Spectrum Disorders: Associations with child characteristics. Journal of Autism and Developmental Disorders. 2008; 38(7):1278. [DOI:10.1007/s10803-007-0512-z] [PMID]
[14] Saberi J, Ghmarane A, Yarmohamadian A. The effectiveness of group positive parenting program on parental stress of mothers of children with autism disorder. Knowledge \& Research in Applied Psychology 2014; 15(2):69-77. http://jsr-p.khuisf.ac.ir/articl e_533919_8e06f0ac6b8ecb9999c06f1aa72be57a.pdf

[15] Rabiee Kenari F, Solgi M. Effectiveness of Resilience Training on Reduction of Parental Stress of Autistic Childrens Mothers. Journal of Ilam University of Medical Sciences. 2015; 23(4):95-105. https://www.sid.ir/en/Journal/ViewPaper.aspx?ID=483037

[16] Mohtashami A, Chimeh N. , Ali Pour Ahmad, Ofoqi Hale. Effects of Training pivotal response treatment on family function in mothers of children with autism. Journal of Family Research . 2012; 8(31): 273-89. https://www.sid.ir/en/journal/ViewPaper. aspx?id $=275474$

[17] Rezaee H, Younesi J, Farahbod M, Ranjbar M. Determining the effectiveness of a modulated parenting skills program on reducing autistic symptoms in children and improvement of parental adjustment. Iranian Rehabilitation Journal. 2018; 16(1):35-44. [DOI:10.29252/nrip.irj.16.1.35]

[18] Esbati M. [The state of deterministic thinking among mothers of Autistic children (Persian)]. Iranian Rehabilitation Journal. 2011; 9(2):10-3. http://irj.uswr.ac.ir/article-1-260-fa.html

[19] Rafati F, Pourmohamadreza-Tajrishi M, Pishyareh E, Mirzaei H, Biglarian A. [Effectiveness of group play therapy on the communication of 5-8 years old children with high functioning $\mathrm{Au}-$ tism (Persian)]. Archives of Rehabilitation. 2016; 17(3):200-11 [DOI:10.21859/jrehab-1703200]

[20] O'Connor KJ, Braverman LM. Play therapy theory and practice: A comparative presentation. Hoboken: John Wiley \& Sons; 1997.

[21] Guerney L. Filial therapy into the $21^{\text {st }}$ century. International Journal of Play Therapy. 2000; 9(2):1-17. [DOI:10.1037/h0089433]

[22] Kaduson HG. Release Play Interventions for Children Who Experienced Stressful Life Events. In: Drewes AA, Schaefer CE. Play-based Interventions for Childhood Anxieties, Fears, and Phobias. New York City: The Guilford Press; 2018. https:// www.guilford.com/books/Play-Based-Interventions-ChildhoodAnxieties-Fears-Phobias/Drewes-Schaefer/9781462534708/ contents

[23] Landreth GL, Bratton SC. Child parent relationship therapy (CPRT): A 10-session filial therapy model. New York City: Taylor \& Francis; 2005. [DOI:10.4324/9780203956342]

[24] VanFleet R, Ryan SD, Smith SK. Filial therapy: A critical review. In: Reddy LA, Files-Hall TM,Z Schaefer CE, editors. Empirically Based Play Interventions for Children. Washington, D.C.: American Psychological Association; 2005. [DOI:10.1037/11086-012]

[25] Kellam T. the parent survival guide: from choas to harmony in ten weeks or less. Tehran: Danzheh; 2015.

[26] Bratton SC, Ray D, Rhine T, Jones L. The efficacy of play therapy with children: A meta-analytic review of treatment outcomes. Professional Psychology: Research and Practice. 2005; 36(4):37690. [DOI:10.1037/0735-7028.36.4.376]

[27] Sue B. Child parent relationship therapy: A review of Controlledoutcome research: The evidence-base for effective practice. Hoboken: Wiley; 2010. [DOI: 10.1002/9781118269626.ch15] 
[28] Dumas JE, Wolf LC, Fisman SN, Culligan A. Parenting stress, child behavior problems, and dysphoria in parents of children with autism, Down syndrome, behavior disorders, and normal development. Exceptionality: A Special Education Journal. 1991; 2(2):97-110. [DOI:10.1080/09362839109524770]

[29] Abidin RR. Parenting stress index-short form. Charlottesville, VA: Pediatric Psychology Press; 1990. [DOI:10.1037/t02445-000]

[30] Fadayi Z, Tahmasiyan K, Farhadi F. [Investigate the factor structure, reliability and validity of short form - Parenting Stress Index mothers of normal children 12-7 years (PSI-SF) (Persian)]. Behavioral Sciences Research. 2010; 2(2):81-91. http:/ /ensani.ir/ file/download/article/20120623092829-8094-11.pdf

[31] Ahmadi SJ, Safari T, Hemmatian M, Khalili Z. [Psychometric Properties of the Autism diagnostic test article (GARS) (Persian)]. Cognitive and Behavioral Sciences. 2011; 1(1):87-104. http://cbs. ui.ac.ir/article_17282_1a9454b924308ae195c549dd9fd2ab4b.pdf

[32] Mirzaie H, Hassani Mehraban A, Hosseini SA, Ghasemi Fard F, Jafari Oori M. Comparison of the effect of filial and adlerian play therapy on attention and hyperactivity of children with attention deficit hyperactivity disorder: A randomized clinical trial. Iranian Rehabilitation Journal. 2019; 17(4):341-50. [DOI: 10.32598/ irj.17.4.341]

[33] Nejati A, Mosavi R, Roshan R, Mahmodigharaei J. [Effectiveness of Child-Parent Relationship Therapy (CPRT) on the improvement of mothers' parenting of children 8 - 11 years with ADHD (Persian)]. Journal of Clinical Psychology. 2016; 8(1):3749. [DOI:10.22075/JCP.2017.2225]

[34] Mohammadi M. [Effectivness intervention educationing mothers and their children with autism on children's social skills 2016 (Persian)]: Paper presented at: The $3^{\text {rd }}$ Scientific Conference on Educational Sciences and Psychology of Social and Cultural Injuries in Iran. 5 Jan 2016; Qom, Iran. https://civilica.com/ doc/446135/

[35] Moqavam F, Nasiriyan M, Mahmodabad Zareyi H. [The effectiveness of parent-child relation therapy on the stress, anxiety, and depression of mothers with a mild mental disability child (Persian)]. Journal of Mazandaran University of Medical Sciences (JMUMS). 2017; 27(151):193-7. http://jmums.mazums.ac.ir/ article-1-5132-fa.pdf

[36] Beckloff DR. Filial therapy with children with spectrum pervasive developmental disorders. Texas: University of North Texas; 1997. 